\title{
Manejo multidisciplinario en el tratamiento integral en odontología. Informe de caso
}

\author{
Multidisciplinary management on the integral treatment in dentistry. \\ Case report
}

\section{Resumen}

Es sumamente importante el manejo multidisciplinario en odontología, para planificar, diagnosticar y establecer un plan de tratamiento adecuado para el beneficio y la satisfacción de los pacientes. El objetivo del caso fue lograr un correcto funcionamiento del sistema masticatorio y a la vez devolver a la paciente la estética pérdida a través del manejo odontológico multidisciplinario. Paciente femenina de 60 ańos, acudió a la Facultad de Odontología de la Universidad Nacional Mayor de San Marcos porque presentaba dificultad para masticar sus alimentos. Al examen clínico se observó espacios entre los dientes anterosuperiores, corona en mal estado, ausencia de piezas dentarias. Radiográficamente se observó que la pieza 48 no ha erupcionado. Entre los principales diagnósticos del paciente tenemos: Pieza dentaria retenida, diastemas entre los dientes anterosuperiores, edéntulo parcial. Se realizó múltiples tratamientos: Exodoncia, restauraciones metal cerámicas, prótesis parcial removible. En conclusión el manejo multidisciplinario en odontología conlleva obtener resultados favorables brindando un enfoque más amplio de las posibilidades de tratamiento para los pacientes.

Palabras clave: Estética sistema masticatorio restauraciones metal cerámicas.

\begin{abstract}
It is extremely important the multidisciplinary management of treatment in dentistry, to diagnose and establish an appropriate treatment plan for the patient benefit and satisfaction. The aim of this case was to achieve a proper functioning of the masticatory system while restoring the patient aesthetics, through a multidisciplinary dental management. A female patient 60, attended the School of Dentistry at Universidad Nacional Mayor de San Marcos because she had difficulty chewing her food. Clinical examination revealed diastema between the upper front teeth, crowns in disrepair and partial absence of teeth. Radiographically it was observed that the piece 48 has not erupted. Additionally has a tooth withheld. Extractions, metal ceramic restorations, removable partial dentures, multiple treatments were performed. In conclusion the multidisciplinary management in dentistry leads to favorable results providing broader treatment possibilities for patients approach.
\end{abstract}

Key words: Aesthetics; masticatory system; metal ceramic restorations.

\section{Introducción}

Para lograr el éxito en la realización de cualquier tratamiento odontológico integral es de suma importancia realizar una planificación ordenada y secuencial del caso, de esta forma se llegará a un correcto diagnóstico que es la base para poder alcanzar el éxito ${ }^{1,2}$. Para ello es fundamental un manejo multidisciplinario, para obtener todas las perspectivas necesarias y así realizar un correcto plan de tratamiento, de esta manera se logrará optimizar los resultados para el beneficio y la satisfacción de los pacientes $^{1,2}$. La visión limitada y enfocada a una sola especialidad puede llevar a no apreciar soluciones clínicas adecuadas en la resolución de los tratamientos ${ }^{2}$.

Los dientes anterosuperiores son uno de los principales elementos al mo- mento de realizar un análisis estético de esta región, y para lograr una apariencia atractiva de las restauraciones, se requiere armonía con toda la apariencia facial $^{3}$. Las restauraciones o coronas de metal cerámica han sido utilizadas con buenos resultados tanto en el sector anterior como posterior desde que fueron introducidas en las restauraciones protésicas, debido a la excelente biocompatibilidad y a la buena estética que presentan su uso es muy frecuente en la práctica clínica diaria, sus usos e indicaciones en odontología han sido ampliamente descritos y son considerados como una buena opción en rehabilitación oral, debido a que cuentan con una alta predictibilidad en los resultados a largo plazo ${ }^{4,5}$.

El requerimiento de la rehabilitación protésica por parte del paciente hace
Caso Clínico

\section{Facultad de Odontología de la Universidad Nacional Mayor de San Marcos, Perú.}

Correspondencia:

C.D. Miguel Cabello Chávez

Jr. Francisco de Zela 1741, Lima 14, Perú. Correo electrónico: angelchavezangel11@hotmail.com
Recibido: 13-04-15

Aceptado: 30-10-15 que el profesional ofrezca diferentes alternativas de tratamiento, para que el paciente pueda escoger el adecuado según su necesidad y economía ${ }^{1}$. La rehabilitación protésica con coronas de metal cerámica combinada con prótesis parcial removible superior e inferior brinda al paciente una alternativa de tratamiento y la solución a su problema bucal de manera eficaz tanto funcional como estética, devolviendo así la función al sistema masticatorio ${ }^{1}$. Es importante mencionar que el uso del articulador semiajustable en rehabilitaciones con prótesis fija combinada con prótesis parcial removible es un instrumento de ayuda para el tratamiento oclusal, permite analizar la relación oclusal entre las piezas dentarias antagonistas y dan información de cómo las articulaciones temporomandibulares se relacionan con la oclusión dentaria ${ }^{6}$. 
El objetivo del presente caso clínico fue lograr un correcto funcionamiento del sistema masticatorio y, a la vez, devolver a la paciente la estética perdida a través del manejo odontológico multidisciplinario.

\section{Reporte del caso}

Figura 1. Exploración clínica y exámenes complementarios.
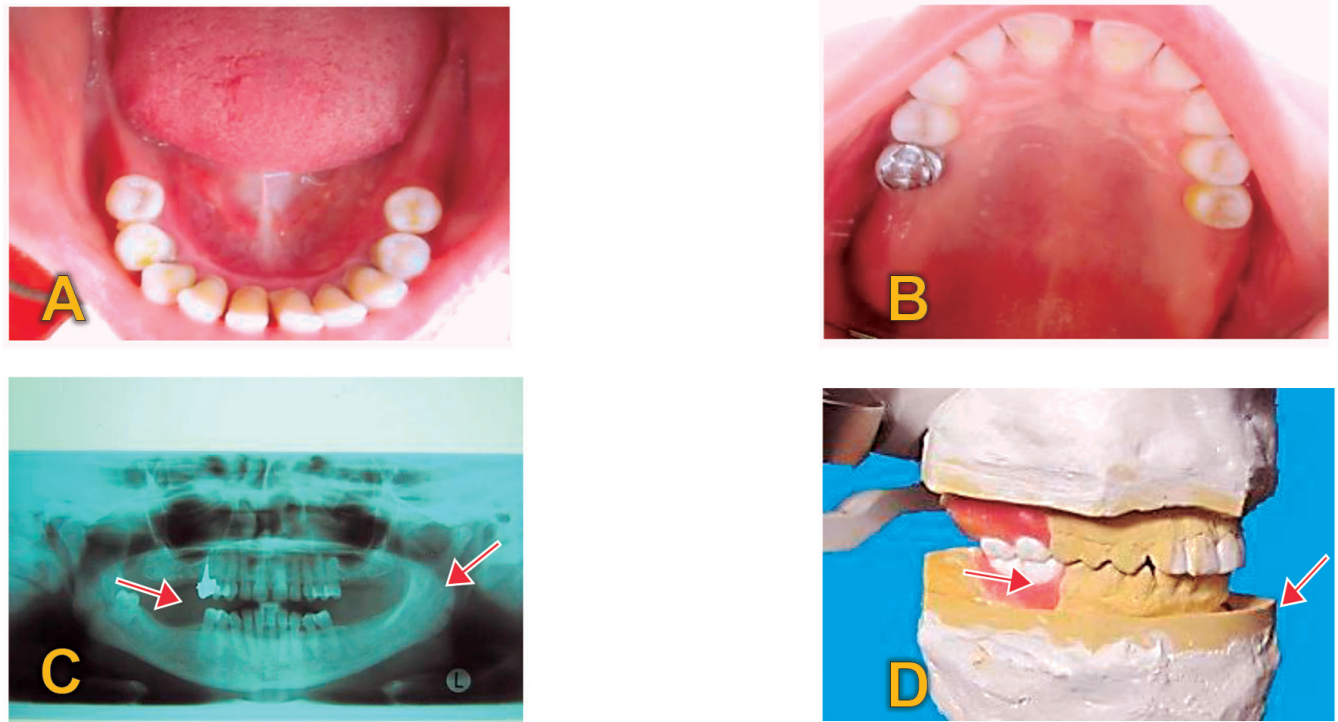

Fig. 1 A: Vista oclusal superior, presencia de diastemas entre los dientes. B: Vista oclusal inferior. C: Radiografía panorámica se observa pieza dentaria 48 retenida y una masa ósea prominente a nivel de la tuberosidad derecha. D) Encerado de diagnóstico en el articulador semiajustable, se observa cierre de los diastemas en el sector anterosuperior y una oclusion posterior estable.

Figura 2. Rehabilitación con coronas metal cerámicas del sector anterosuperior
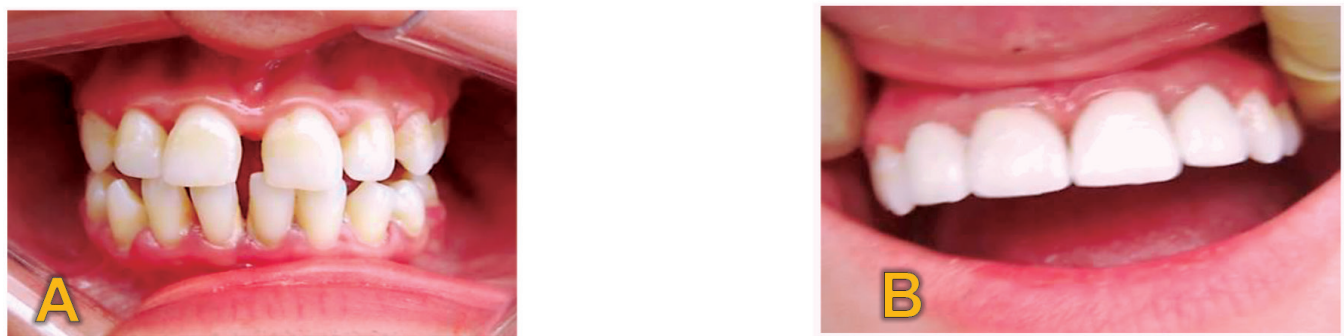

Fig. 2 A: Vista inicial con presencia de diastemas entre las piezas dentarias 13, 12, 11, 21, 22 y 23. B: Cementación definitiva de las coronas metal porcelana.

Figura 3. Rehabilitación con prótesis parcial removible del sector posterior superior e inferior.
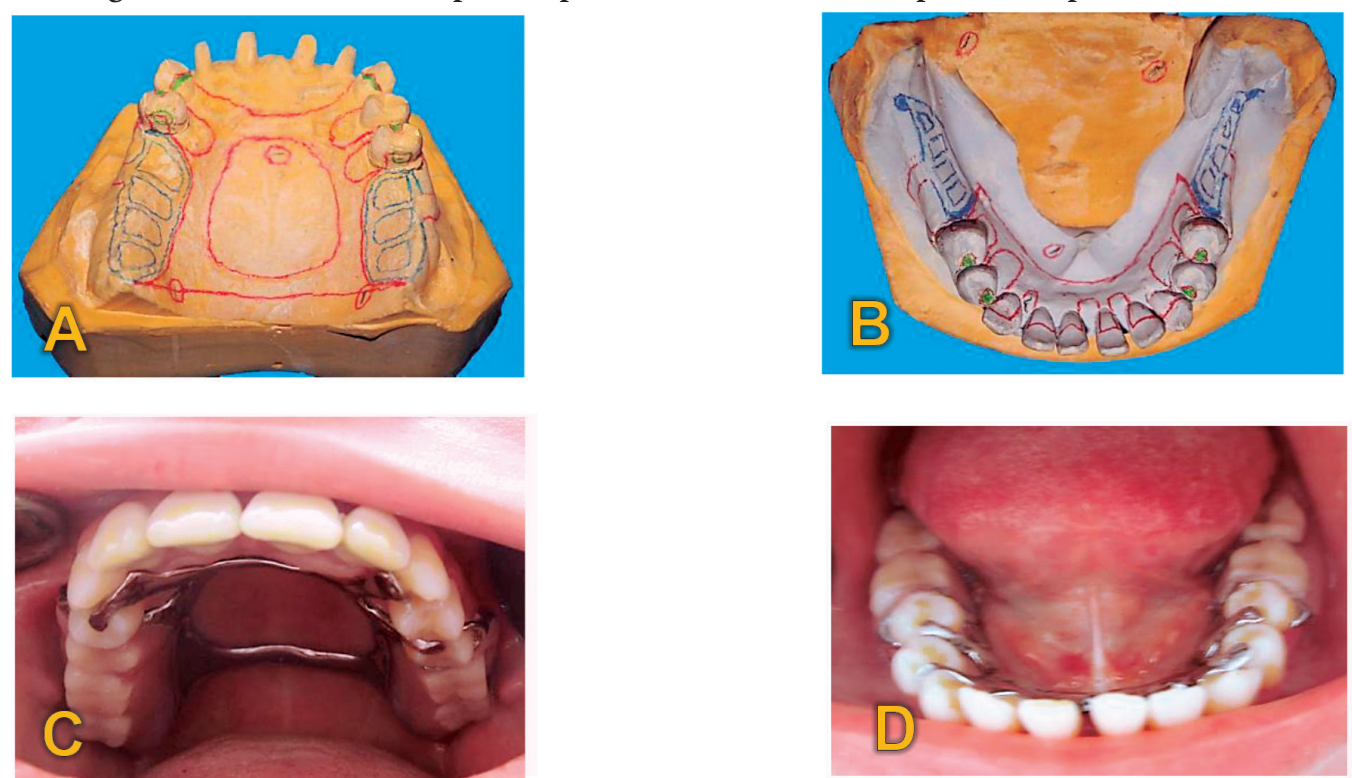

Fig. 3 A: Diseño de la prótesis parcial removible en el modelo superior. B: Diseńo de la prótesis parcial removible en el modelo inferior. C y D: Prótesis parcial removible superior e inferior instalada en boca. 
Paciente de sexo femenino de 60 años de edad, de raza mestiza, acudió a la clínica de la Facultad de Odontología de la Universidad Nacional Mayor de San Marcos expresando dificultad para masticar sus alimentos y no le gusta el aspecto de sus dientes anteriores. La dieta de la paciente básicamente estuvo dada por alimentos blandos debido a la gran ausencia de piezas dentarias.

A la exploración clínica se observó lesiones cariosas; corona en mal estado; espacios entre los dientes anterosuperiores; ausencia de piezas dentarias. En la radiografía panorámica se observó que la pieza 48 no ha erupcionado y una masa ósea prominente a nivel de la tuberosidad derecha. Los principales diagnósticos fueron: Gingivitis asociada a placa dental, pieza 48 retenida, corona de Venner desadaptada en pieza 15, caries dental, diastemas entre los dientes anterosuperiores, edéntulo parcial superior e inferior. El plan de tratamiento consistió en hacer: 1) Fisioterapia y profilaxis. 2) Exodoncia de la pieza 15 , 48 y remodelación de la tuberosidad derecha. 3) Restauraciones directas con resina en las piezas: $14,24,25$ y 44. 4) Coronas metal cerámicas en piezas 1222. 5) Prótesis parcial removible superior e inferior (Fig. 1).

Se inició el tratamiento multidisciplinariamente con el consentimiento informado del paciente. Se realizó una profilaxis bucal, se retiró la placa dental, lográndose inicialmente la antisepsia a nivel bucal. Se instruyó a la paciente para una correcta higiene bucal.

Se realizó la exodoncia de la pieza dentaria retenida 48 y de la pieza 15 debido a que presentó pobre soporte óseo radicular para ser considerada como pilar de la prótesis parcial removible; también se realizó una remodelación de la tuberosidad derecha, se esperó en promedio 3 meses para que las zonas operadas cicatricen y se fueron restaurando áreas alejadas.

Se restauraron con resina (3M, Estados Unidos) por oclusal las piezas dentarias 14, 24, 25 y 44 .

Se realizaron preparaciones dentarias para las coronas metal porcelana en las piezas 12-22 y se adaptaron las coronas provisonales elaboradas según el encerado de diagnóstico. En la siguiente cita se procedió la impresión definitiva mediante la técnica de las copas de Ripol y el rebase con alginato (Zhermack, Italia), se tomó registro intermaxilar y se envió al laboratorio para la elaboración de las cofias metálicas. Estas se proba- ron en boca y se seleccionó el color de las coronas. Se realizó el glaseado de las coronas y se verificó el color de las piezas y la oclusión dentaria en boca, posteriormente se procedió a la cementación definitiva (Fig.2).

Para la confección de la prótesis parcial removible, se hizo el diseño de la base metálica, se realizó la preparación biostática y se tomó la impresión a la arcada superior e inferior. Se tomó el registro intermaxilar y se seleccionó el color de los dientes. Luego se procedió a la prueba del enfilado de los dientes en boca, verificando el color y la oclusión dentaria; posteriormente se instaló definitivamente las prótesis en la boca del paciente. Se instruyó al paciente acerca del mantenimiento e higiene de las prótesis y de sus tejidos remanentes. Se citó a la semana, luego al mes, a los tres meses, y finalmente cada seis meses (Fig.3).

\section{Discusión}

La interrelación con otras disciplinas o el tratamiento multidisciplinario en odontología nos lleva a planificar de una mejor manera las diversas situaciones clínicas que se presentan en la práctica clínica diaria; de esta forma integramos a las diferentes especialidades para ofrecer mejores alternativas de tratamiento y obtener una mayor satisfacción en los pacientes ${ }^{2,7}$.

Las coronas metal cerámicas son frecuentemente utilizadas en la práctica clínica habitual en odontología, debido a que estas presentan una excelente biocompatibilidad y buena estética ${ }^{5}$. A pesar de la evolución mecánica de las estructuras cerámicas en situaciones clínicas de rehabilitaciones de prótesis parcial fija extensa no hay un seguimiento que demuestre su éxito en un periodo a largo plazo $^{8}$, pero existen tratamientos de casos extensos con coronas metal cerámicas, donde el uso de las estas está más indicado al presentar mayor resistencia a la fractura en un porcentaje de 5 a $10 \%$ en 10 ańos de permanencia en boca ${ }^{9,10}$. En caso de fracturas es importante mencionar que una de las ventajas de las coronas de metal cerámicas es la reparación directa o indirecta en prótesis parcial fija, es una alternativa bastante viable debido al bajo costo con relación a una nueva rehabilitación, al tiempo clínico-laboratorial reducido y a la facilidad de ejecución del trabajo ${ }^{11,12}$.

La combinación entre una prótesis parcial fija y removible resulta ser una buena alternativa de tratamiento tanto funcional como estética, de esta forma el profesional ofrece diferentes alternativas de tratamiento para que el paciente pueda escoger el adecuado, según su necesidad y economía ${ }^{1}$.

El tiempo de cicatrización después de la cirugía es esencial en el desarrollo del tratamiento protésico, debido a que un adelanto en el tiempo de la toma de la impresión definitiva podría traer consecuencias indeseables en los resultados finales de las restauraciones protésicas ${ }^{13}$.

\section{Conclusión}

El tratamiento integral en odontología mediante la integración con las diferentes especialidades nos lleva a obtener resultados clínicos favorables, de esta forma tenemos un enfoque más amplio de las diferentes alternativas de tratamiento en los pacientes. El profesional debe trabajar multidisciplinariamente para identificar las patologías del sistema masticatorio, planificar adecuadamente el caso clínico y realizar un correcto diagnóstico, que es la base para poder alcanzar el éxito, y de esta forma establecer un óptimo plan de tratamiento para el beneficio del paciente. La rehabilitación oral con coronas de metal cerámica combinada con prótesis parcial removible bien planificadas y correctamente confeccionadas ofrece a los pacientes una buena alternativa de tratamiento y una solución eficaz tanto funcional como estética.

\section{Referencias bibliográficas}

1. Blanco F, Abreu E. Prótesis híbrida: presentación de caso. Rev Méd Electrón. 2011;33(5);626-32.

2. Villaseñor C, López M. Tratamiento interdisciplinario de paciente con sonrisa gingival: Reporte de un caso. Rev Odont Mex. 2013;17(1):51-6.

3. Cabello M, Alvarado S. Relationship between the shape of the upper central incisors and the facial contour in dental students. Lima. Peru. J Oral Res 2015; 4(3): 189-6.

4. Andreatta O, Nishioka R. Avaliação da resistência à tração entre sistemas para reparo de revestimento estético e liga de níquel-cromo. Braz Dent Sci. 2011; 14(4):49-59.

5. Ozcan M. Evaluation of alternative intra-oral repair techniques for fractured ceramic-fused-tometal restorations. J Oral Rehabil. 2003;30:194-203. 
6. Okeson J. Oclusion Y afecciones temporomandibulares. $6^{\mathrm{a}}$ ed. Barcelona: Mosby/Doyma; 2008.

7. Lamas C, Paz J, Paredes G, Angulo G, Cardozo S. Rehabilitación Integral en Odontología, Odontol Sanmarquina 2012;15(1):31-4.

8. Vilarrubí A, Pebé P, Rodríguez A. Prótesis fija convencional libre de metal: tecnología CAD CAMZirconia, descripción de un caso clínico. Odontoestomatología 2011;13(18):16-28
9. Ozcan M. Fracture reasons in ceramic-fused-to-metal restorations. J Oral Rehabil 2003;30(3):265-9.

10. Siegward D, Heintze D. Survival of zirconia and metal-supported fi xed dental prostheses: a systematic review. Int $\mathrm{J}$ Prosthodont. 2010;23(6):493-2.

11. Bravo D, Bustamante E, Daza V. Influencia del tratamiento de superficie en la resistencia traccional de porcelana feldespática reparada con resina compuesta. Rev Clin
Periodoncia Implantol Rehabil Oral. 2014;7(3):123-7

12. Fraxino B, Goulart C, Caregnatto M, Pedroso L, Leite M. Técnica Indirecta Para Reparación De Prótesis Parcial Fija Metal-Porcelana Relato Del Caso. Acta odontol venez. 2010;48(1):164-9.

13. Agudelo C, Rico J, Molano E. Alargamiento coronal preprotésico: un procedimiento estético y funcional. Reporte de un caso. Rev Estomat. 2010;18(2):23-2. 